\title{
Medical Image Fusion Algorithm based on Adaptive Selectivity Reconstruction and Pulse Coupled-Neural Network
}

\author{
Mohamed El Aallaoui \\ Department of Statistics and Applied Mathematics in Economics \\ FSJES Aïn Sebaâ, Hassan II University, Casablanca Morocco
}

\begin{abstract}
We propose a novel medical image fusion framework, based on adaptive selectivity reconstruction and pulse coupled-neural network. The proposed method takes advantage of the multiscale analysis and multiselectivity analysis, which enables it to capture the different structures information of different modality medical images. A subjective assessment comparing our method and other medical image fusion methodes is performed. Experiments showed that our method is more robust than the others, in both visual effect and objective evaluation criteria.
\end{abstract}

\section{Keywords}

Continuous Wavelet Transform; Directional Wavelet; Angular Selectivity; Adaptive Selectivity; Medical Image Fusion.

\section{INTRODUCTION}

Over the past few decades, the development of medical technology, computer technology, and biomedical engineering has given a variety of medical images for clinical diagnosis. These medical images with different modalities focus on different categories of organ / tissue information. For example, computed tomography (CT) imaging scan accurately detects dense structures such as bones. While magnetic resonance imaging (MR) provides high-resolution anatomical information for soft tissues, it is less sensitive for diagnosing dense structures than computed tomography. In addition to these anatomical imaging techniques, PET, SPECT, and functional magnetic resonance imaging (fMRI) provide functional information, such as an organism's metabolism, but often the spatial resolution of these functional images is very low [1, 2].

Reliance on a single medical image does not provide a comprehensive and accurate clinical diagnosis. Therefore, various medical images are combined to provide much more useful information through the image fusion of medical images, which aims to create a composite image to combine various information of different medical images. Many approaches have been designed for addressing this task in recent years, these approaches can be classified in two different ways: medical image fusion in spatial domain [3, 4, 5] and medical image fusion in the multi-scale transform (MST) Domain [6] 7].

In general, medical image fusion schemes based on multi-scale transforms comprise three steps. First, the source images are con- verted into an MST domain. Then, the multi-scale representations of the source images are fused a using pulse couple neuron network (PCNN). Finally, the fused image is acquired from the merged coefficients by performing the inverse transform.

MST approaches hat are commonly used in image fusion, include discrete wavelet transform [8], Laplacian pyramid (LP) [9], contourlet transform [10], nonsubsampled contourlet transform (NSCT) and nonsubsampled shearlet transform (NSST) [11, 12]. NSCT and NSST inherit the perfect properties of contourlet and LP, such as the characteristics of timefrequency localization, multidirection, and anisotropy, and meanwhile possesses the shiftinvariance which contourlet transform lacks of, leading to better frequency selectivity and regularity than contourlet transform and get rid of pseudo-Gibbs phenomena along the edges to some extent [1, 13].

Medical image are not simple images characterized by their types of edges and contours oriented ones, like straight edges, to more isotropic objects, like corners and spots. Between these two extreme behaviors, we find, for instance, curves and texture points. Each of these objects is well represented by wavelets with different angular selectivity. However, since in LP, NSCT and NSST the wavelets share the same angular selectivity, isotropic, directional and less-oriented features are processed under the same framework with the same angular selectivity. In order to overcome this limitation of representation of geometric structure in thise medical image fusion methods, we have developed a new shift-invariant adaptive selectivity representation which can represent the different structures of the medical image.

The paper is organized as follows. In section 2, we recall the frame formalism of the 2-D continuous wavelet transform. In section 3, we propose a shift-invariant multiscale frame. In section 4 we propose a new representation of image and we will how this representation can provide a new degree of freedom to describe adaptively the different structures of the image geometry. We recall The pulsecoupled neural network in section 5. Our algorithm for medical image fusion is described in the section 6 . We report the results of our experiments in section 7 and conclude the paper in section 8 .

\section{2-D CONTINUOUS WAVELET TRANSFORM}

In order to fix the notations, we first recall the basic formulas. Let $f \in L^{2}\left(\mathbb{R}^{2}\right)$ be an image of finite energy. Given an admissible wavelet $\psi \in L^{1}\left(\mathbb{R}^{2}\right) \cap L^{2}\left(\mathbb{R}^{2}\right)$, the continuous wavelet transform 
of $f$ is the function 14,15

$$
\begin{aligned}
w_{\vec{b}, s, \theta} & =\left\langle\psi_{\vec{b}, s, \theta} \mid f\right\rangle \\
& =s^{-2} \int_{\mathbb{R}^{2}} \psi^{*}\left(r_{\theta}^{-1} \frac{\vec{x}-\vec{b}}{s}\right) f(\vec{x}) d^{2} \vec{x} \\
& =\frac{1}{(2 \pi)^{2}} \int_{\mathbb{R}^{2}} \widehat{\psi}^{*}\left(s r_{\theta}^{-1} \vec{k}\right) \widehat{f}(\vec{k}) e^{i \vec{k} \cdot \vec{b}} d^{2} \vec{k}
\end{aligned}
$$

where $\psi_{\vec{b}, s, \theta}$ is copy of $\psi$, translated by $\vec{b} \in \mathbb{R}^{2}$, dilated by $s \in \mathbb{R}_{+}^{*}$ and rotated by $\theta \in S_{1} \simeq[0,2 \pi)$.

$$
r_{\theta}^{-1}\left(\begin{array}{cc}
\cos \theta & \sin \theta \\
-\sin \theta & \cos \theta
\end{array}\right)
$$

and the hat denotes the standard Fourier transform on $L^{2}\left(\mathbb{R}^{2}\right)$ When the aim is to detect oriented features in an image (for instance, in the classical problem of edge detection or in directional filtering), one has to use a directional wavelets. That is, a wavelet $\psi \in L^{2}\left(\mathbb{R}^{2}\right)$ whose Fourier transform $\widehat{\psi} \in L^{2}\left(\mathbb{R}^{2}\right)$ has its numerical support contained in a convex cone with apex at the origin in Fourier space. A directional wavelet is characterized by its angular selectivity (or Angular Resolving Power), that is, its ability to distinguish features with close orientations. This quantity is by definition inversely proportional to the aperture of the support cone [14, 15, 16, 17].

The half-continuous frame theory addresses the issue of reconstructing an signal $f$ from sample coefficients of a particular transform. For 2-D continuous wavelet transform, the discretization of parameters $s$ and $\theta$ while preserving a perfect reconstruction formula. Let $\Gamma=\left\{\left(\vec{b}, s_{j}, \theta_{n}\right): \vec{b} \in \mathbb{R} ; j \in \mathbb{Z} ; n=0,1, \ldots, N-1\right\}$ be a half-continuous grid, where $s_{j}=2^{j}$ and $\theta_{n}=n \frac{2 \pi}{N}$. If

$$
\sum_{j \in \mathbb{Z}} \sum_{n=0}^{N-1}\left|\widehat{\psi}\left(s_{j} r_{\theta_{n}}^{-1} \vec{k}\right)\right|^{2}=1, \quad \forall \vec{k} \in \mathbb{R}^{2}-\{\overrightarrow{0}\},
$$

then the family $\left\{\psi_{\vec{b}, s_{j}, \theta_{n}}:\left(\vec{b}, s_{j}, \theta_{n}\right) \in \Gamma\right\}$ is a tight halfcontinuous frame of $L^{2}\left(\mathbb{R}^{2}\right)$, and the following reconstruction formula holds strongly in $L^{2}\left(\mathbb{R}^{2}\right)$, [14, 18]

$$
f(\vec{x})=\sum_{j \in \mathbb{Z}} \sum_{n=0}^{N-1} \int_{\mathbb{R}^{2}}\left\langle\psi_{\vec{b}, s_{j}, \theta_{n}} \mid f\right\rangle \psi_{\vec{b}, s_{j}, \theta_{n}}(\vec{x}) d^{2} \vec{b}
$$

\section{SHIFT-INVARIANT MULTISCALE FRAME}

Let $h$ be a pair of finite impulse response filter in 1-D. Suppose that $h$ is a low-pass filter whose transfer function satisfies $\widehat{h}(0)=$ $\sqrt{2}$. In 2-D, we construct a pair of filters $(H, G)$ whose transfer functions are

$$
\widehat{H}(\vec{k})=\widehat{h}\left(k_{1}\right) \widehat{h}\left(k_{2}\right)
$$

and

$$
\widehat{G}(\vec{k})=e^{i \vec{k}} \sqrt{|\widehat{H}(\overrightarrow{0})|^{2}-|\widehat{H}(\vec{k})|^{2}},
$$

where $\vec{k}=\left(k_{x}, k_{y}\right)$. As $\widehat{h}$ is a 1-D low-pass finite impulse response filter, the equations (7) and (8) imply the filter $H$ is 2D low-pass finite impulse response filter, and the filter $G$ is $2 \mathrm{D}$ bas-pass finite impulse response filter. We consider the infinite products

$$
\prod_{p=1}^{+\infty} \frac{\widehat{H}\left(2^{-p} \vec{k}\right)}{2}=\prod_{p=1}^{+\infty} \frac{\widehat{h}\left(2^{-p} k_{x}\right)}{\sqrt{2}} \prod_{p=1}^{+\infty} \frac{\widehat{h}\left(2^{-p} k_{y}\right)}{\sqrt{2}}
$$

As $h$ a low-pass filter, the condition $\widehat{h}(0)=\sqrt{2}$ implies

$$
\left|\widehat{h}\left(k_{x}\right)\right| \leq|\widehat{h}(0)|=\sqrt{2} \leq \sqrt{2} e^{\left|k_{x}\right|}
$$

hence

$$
\prod_{p=1}^{+\infty} \frac{\widehat{h}\left(2^{-p} k_{x}\right)}{\sqrt{2}} \leq \prod_{p=1}^{+\infty} e^{2^{-p}\left|k_{x}\right|} \leq e^{\sum_{p=1}^{+\infty}\left(2^{-p}\left|k_{x}\right|\right)} \leq e^{\left|k_{x}\right|}
$$

Therefore the infinite product $\prod_{p=1}^{+\infty} \frac{\widehat{H}\left(2^{-p} \vec{k}\right)}{2}$ converge pointwise. In 2-D, we construct two functions $\phi$ and $\phi$ whose Fourier transforms are

$$
\widehat{\phi}(\vec{k})=\prod_{p=1}^{+\infty} \frac{\widehat{H}\left(2^{-p} \vec{k}\right)}{2}=\frac{1}{2} \widehat{H}\left(\frac{\vec{k}}{2}\right) \widehat{\phi}\left(\frac{\vec{k}}{2}\right)
$$

and

$$
\widehat{\psi}(\vec{k})=\frac{1}{2} \widehat{G}\left(\frac{\vec{k}}{2}\right) \widehat{\phi}\left(\frac{\vec{k}}{2}\right),
$$

As $H$ and $G$ are finite impulse response filters, the equations (12) and $(13)$ imply that $\widehat{\phi}$ and $\widehat{\psi}$ are finite energy functions [19] so that $(\phi, \psi) \in L^{2}\left(\mathbb{R}^{2}\right) \times L^{2}\left(\mathbb{R}^{2}\right)$.

Since the filter $H$ is 2D low-pass finite impulse response filter and the filter $G$ is $2 \mathrm{D}$ bas-pass finite impulse response filter, the the function $\phi$ is a scaling function which captures low frequencies, against $\psi$ is a wavelet which captures high frequencies.

Let $\psi_{\vec{b}, j}$ be the wavelets $\psi$ translated by $\vec{b} \in \mathbb{R}^{2}$, dilated by $2^{j}$

$$
\psi_{\vec{b}, j}(\vec{x})=\frac{1}{2^{2 j}} \psi\left(\frac{\vec{x}-\vec{b}}{2^{j}}\right)
$$

We also use the notion

$$
\psi_{j}(\vec{x})=\frac{1}{2^{2 j}} \psi\left(\frac{\vec{x}}{2^{j}}\right) .
$$

Thus the Fourier transform of $\psi_{\vec{b}, j}$ is defined by

$$
\widehat{\psi}_{\vec{b}, j}(\vec{k})=\widehat{\psi}\left(2^{j} \vec{k}\right) e^{i \vec{k} \vec{b}}
$$

The continuous wavelet transform of the function $f \in L^{2}\left(\mathbb{R}^{2}\right)$ with wavelets $\psi_{\vec{b}, j}$ is

$$
\begin{aligned}
w_{\vec{b}, j} & =\left\langle\psi_{\vec{b}, j} \mid f\right\rangle \\
& =\int_{\mathbb{R}^{2}} \psi_{\vec{b}, j}^{*}(\vec{x}) f(\vec{x}) d^{2} \vec{x} \\
& =\int_{\mathbb{R}^{2}} \psi_{j}^{*}(\vec{x}-\vec{b}) f(\vec{x}) d^{2} \vec{x} \\
& =\psi_{j} \star f(\vec{b})
\end{aligned}
$$

Therefore, the continuous wavelet transform can be rewritten as a convolution product $\psi_{j} \star f(\vec{b})$, then the convolution theorem of Fourier transform imply that

$$
\widehat{w}_{., j}(\vec{k})=\widehat{\psi}_{j}(\vec{k}) \widehat{f}(\vec{k})=\widehat{\psi}\left(2^{j} \vec{k}\right) \widehat{f}(\vec{k})
$$


Moreover the continuous wavelet transform is translation-invariant, Indeed, if $f_{\vec{u}}(\vec{x})=f(\vec{x}-\vec{u})$, then

$$
\begin{aligned}
w_{\vec{b}, j}\left(f_{\vec{u}}\right) & =\left\langle\psi_{\vec{b}, j} \mid f_{\vec{u}}\right\rangle \\
& =\int_{\mathbb{R}^{2}} \psi_{\vec{b}, j}^{*}(\vec{x}) f(\vec{x}-\vec{u}) d^{2} \vec{x} \\
& =\int_{\mathbb{R}^{2}} \psi_{j}^{*}(\vec{x}-\vec{b}+\vec{u}) f(\vec{x}) d^{2} \vec{x} \\
& =\left\langle\psi_{\vec{b}-\vec{u}, j} \mid f_{\vec{u}}\right\rangle \\
& =w_{\vec{b}-\vec{u}, j}(f)
\end{aligned}
$$

But a uniform sampling $\vec{b}_{n}$ of the position parameter $\vec{b}$ destroys this invariance, because the continuous wavelet transforms $w_{\vec{b}_{n}, j}\left(f_{\vec{u}}\right)$ and $w_{\vec{b}_{n}-\vec{u}, j}(f)$ can give different input values if $\vec{u} \neq \vec{b}_{n}$.

The following theorem gives a sufficient condition to guarantee that the family $\left.\left.\left\{\phi_{J, \vec{b}}, \psi_{j, \vec{b}}: j \in\right]-\infty, J\right], \vec{b} \in \mathbb{R}^{2}\right\}$ is a shift-invariant frame of $L^{2}\left(\mathbb{R}^{2}\right)$.

THEOREM 1. For any $J \in \mathbb{Z}$ the family $\left\{\phi_{J, \vec{b}}, \psi_{j, \vec{b}}: j \in\right.$ ] $\left.-\infty, J], \vec{b} \in \mathbb{R}^{2}\right\}$ is a tight half-continuous frame for $L^{2}\left(\mathbb{R}^{2}\right)$. Therefore, any function $f \in L^{2}\left(\mathbb{R}^{2}\right)$ is represented as:

$$
f(\vec{x})=\left[\zeta_{., J} \star \phi_{J}\right](\vec{x})+\sum_{j=-\infty}^{J}\left[\omega_{., j} \star \psi_{j}\right](\vec{x}),
$$

with

$$
\begin{gathered}
w_{\vec{b}, j}=\left\langle\psi_{\vec{b}, j} \mid f\right\rangle, \quad \zeta_{\vec{b}, j}=\left\langle\phi_{\vec{b}, j} \mid f\right\rangle \\
\psi_{\vec{b}, j}(\vec{x})=\frac{1}{2^{2 j}} \psi\left(\frac{\vec{x}-\vec{b}}{2^{j}}\right) \quad \text { and } \quad \phi_{\vec{b}, j}(\vec{x})=\frac{1}{2^{2 j}} \phi\left(\frac{\vec{x}-\vec{b}}{2^{j}}\right) .
\end{gathered}
$$

Proof. To prove that the family $\left\{\phi_{J, \vec{b}}, \psi_{j, \vec{b}}: \quad j \in\right]-$ $\left.\infty, J], \vec{b} \in \mathbb{R}^{2}\right\}$ is a tight half-continuous frame for $L^{2}\left(\mathbb{R}^{2}\right)$, it suffices to evaluate the equality:

$$
\left|\widehat{\phi}\left(2^{J} \vec{k}\right)\right|^{2}+\sum_{i=-\infty}^{J}\left|\widehat{\psi}\left(2^{j} \vec{k}\right)\right|^{2}=1 \quad \forall \vec{k} \in \mathbb{R}^{2}-\{\overrightarrow{0}\} .
$$

Let us prove first that

$$
\widehat{G}(\vec{k}) \widehat{G}^{*}(\vec{k})=4-\widehat{H}(\vec{k}) \widehat{H}^{*}(\vec{k}) .
$$

As $|\widehat{h}(0)|=\sqrt{2}$, equation (7) implies

$$
\widehat{H}(\vec{k}) \widehat{H}^{*}(\vec{k})=\widehat{h}(0) \widehat{h}(0) \widehat{h}^{*}(0) \widehat{h}^{*}(0)=|\widehat{h}(0)|^{2}|\widehat{h}(0)|^{2}=4 .
$$

Hence

$$
\begin{aligned}
\widehat{G}(\vec{k}) \widehat{G}^{*}(\vec{k}) & =|\widehat{G}(\vec{k})|^{2} \\
& =\left|e^{i \vec{k}} \sqrt{|\widehat{H}(\overrightarrow{0})|^{2}-|\widehat{H}(\vec{k})|^{2}}\right|^{2} \\
& =|\widehat{H}(\overrightarrow{0})|^{2}-|\widehat{H}(\vec{k})|^{2} \\
& =4-|\widehat{H}(\vec{k})|^{2} \\
& =4-\widehat{H}(\vec{k}) \widehat{H}^{*}(\vec{k}) .
\end{aligned}
$$

We shall now prove that

$$
\widehat{\phi}\left(2^{J} \vec{k}\right) \widehat{\phi^{*}}\left(2^{J} \vec{k}\right)+\sum_{i=-\infty}^{J} \widehat{\psi}\left(2^{j} \vec{k}\right) \widehat{\psi}^{*}\left(2^{j} \vec{k}\right)=1, \forall \vec{k} \in \mathbb{R}^{2}-\{\overrightarrow{0}\}
$$

The Fourier transform expressions (13, , 12) and quality (29) prove that:

$$
\begin{aligned}
\widehat{\psi}(\vec{k}) \widehat{\psi}^{*}(\vec{k}) & =\frac{1}{4} \widehat{G}\left(\frac{\vec{k}}{2}\right) \widehat{G}^{*}\left(\frac{\vec{k}}{2}\right) \widehat{\phi}\left(\frac{\vec{k}}{2}\right) \widehat{\phi}^{*}\left(\frac{\vec{k}}{2}\right) \\
& =\frac{1}{4}\left(4-\widehat{H}(\vec{k}) \widehat{H}^{*}(\vec{k})\right) \widehat{\phi}\left(\frac{\vec{k}}{2}\right) \widehat{\phi}^{*}\left(\frac{\vec{k}}{2}\right), \\
& =\widehat{\phi}\left(\frac{\vec{k}}{2}\right) \widehat{\phi}^{*}\left(\frac{\vec{k}}{2}\right)-\frac{1}{4} \widehat{G}(\vec{k}) \widehat{G}^{*}(\vec{k}) \widehat{\phi}\left(\frac{\vec{k}}{2}\right) \widehat{\phi}^{*}\left(\frac{\vec{k}}{2}\right) \\
& =\widehat{\phi}\left(\frac{\vec{k}}{2}\right) \widehat{\phi}^{*}\left(\frac{\vec{k}}{2}\right)-\widehat{\phi}(\vec{k}) \widehat{\phi}^{*}(\vec{k}) .
\end{aligned}
$$

Hence for $p>0, J>0$,

$\sum_{j=-p}^{J} \widehat{\psi}\left(\left(2^{j} \vec{k}\right) \widehat{\psi}^{*}\left(\left(2^{j} \vec{k}\right)=\widehat{\phi}\left(\left(2^{-p-1} \vec{k}\right) \widehat{\phi}^{*}\left(\left(2^{-p-1} \vec{k}\right)-\widehat{\phi}\left(\left(2^{J} \vec{k}\right) \widehat{\phi}^{*}\left(\left(2^{J} \vec{k}\right)\right.\right.\right.\right.\right.\right.$

As $\widehat{h}(0)=\sqrt{2}$, equation 12 implies $\widehat{\phi}(\overrightarrow{0})=\widehat{\phi}^{*}(\overrightarrow{0})=1$. Letting $p \rightarrow+\infty$ yields

$\widehat{\phi}\left(2^{J} \vec{k}\right) \widehat{\phi}^{*}\left(2^{J} \vec{k}\right)+\sum_{i=-\infty}^{J} \widehat{\psi}\left(2^{j} \vec{k}\right) \widehat{\psi}^{*}\left(2^{j} \vec{k}\right)=1 \quad \forall \vec{k} \in \mathbb{R}^{2}-\{\overrightarrow{0}\}$.

Thus the equality 30 is verified.

\section{ADAPTIVE SELECTIVITY RECONSTRUCTION}

In this section we propose a new Shift-invariant representation which provide a new degree of freedom to describe adaptively the different structures of the image geometry.

We consider $2 \pi$-periodic function $D_{\alpha}$ defined by

$$
D_{\alpha}(\theta)= \begin{cases}1, & \theta \in\left[\frac{\pi}{2}+\alpha, \frac{3 \pi}{2}-\alpha\right] ; \\ 0, & \theta \in\left[0, \frac{\pi}{2}-\alpha\right] \cup\left[\frac{3 \pi}{2}+\alpha, 2 \pi\right] ; \\ \beta\left(\frac{-\pi / 2+\theta}{\alpha}\right), & \theta \in\left[\frac{\pi}{2}-\alpha, \frac{\pi}{2}+\alpha\right] ; \\ \beta\left(\frac{3 \pi / 2-\theta}{\alpha}\right), & \theta \in\left[\frac{3 \pi}{2}-\alpha, \frac{3 \pi}{2}+\alpha\right] .\end{cases}
$$

where the function $\beta$ is defined in $[-1,1]$ by

$$
\beta(t)=\sin \left(\frac{\pi}{4}(t+1)\right),
$$

the function $\beta$ satisfies

$$
\beta^{2}(t)+\beta^{2}(-t)=1 .
$$

For $L \in \mathbb{N}^{*}$ and $\alpha=\frac{\pi}{2^{L}}$, we create $2^{l}$ different functions $\eta_{l, n}$ indexed by $n \in\left\{0, \ldots, 2^{l}-1\right\}$ for any $l \in\{0, \ldots, L\}$ defined by:

$$
\eta_{0,0}(\theta)=1 \text {, }
$$

for $n=0, \ldots, 2^{l}-1$

$$
\eta_{l+1,2 n}(\theta)=\eta_{l, n}(\theta) D_{\alpha}\left(\theta-\frac{(2 n+1) \pi}{2^{l}}\right)
$$

and

$$
\eta_{l+1,2 n+1}(\theta)=\eta_{l, n}(\theta) D_{\alpha}\left(\theta-\frac{(2 n+1) \pi}{2^{l}}-\pi\right)
$$


Proposition 1. For any $l \in\{1, \ldots, L\}$ the parameter $n$ describes a translation of $\theta_{l, n}=n \frac{2 \pi}{2^{l}}$ :

$$
\eta_{l, n}(\theta)=\eta_{l, 0}\left(\theta-\theta_{l, n}\right) \quad n=0,1, \ldots, 2^{l}-1
$$

and the functions $\eta_{l, n}$ have a support of the same size and equal to $\frac{2 \pi}{2^{l}}+2 \alpha$.

Proof. One have for any $l \in\{1, \ldots, L\}$

$$
\begin{gathered}
\eta_{l, 1}(\theta)=D_{\alpha}\left(\theta-\frac{\pi}{2^{l-1}}-\pi\right) \prod_{p=0}^{l-2} D_{\alpha}\left(\theta-\frac{\pi}{2^{p}}\right), \\
\eta_{l, 0}(\theta)=D_{\alpha}\left(\theta-\frac{\pi}{2^{l-1}}\right) \prod_{p=0}^{l-2} D_{\alpha}\left(\theta-\frac{\pi}{2^{p}}\right),
\end{gathered}
$$

By [31, these two functions $\eta_{l, 1}$ and $\eta_{l, 0}$ are related by the translation relation:

$$
\eta_{l, 1}(\theta)=\eta_{l, 0}\left(\theta-\frac{2 \pi}{2^{l}}\right),
$$

and the size of their supports equals to $\frac{2 \pi}{2^{l}}+2 \alpha$.

Suppose that for a fixed $l$ we have:

$$
\eta_{l, n}(\theta)=\eta_{l, 0}\left(\theta-\theta_{l, n}\right), \quad n=0,1, \ldots, 2^{l}-1 .
$$

The properties 35 and 39) imply that

$$
\begin{aligned}
\eta_{l+1,2 n}(\theta) & =\eta_{l, n}(\theta) D_{\alpha}\left(\theta-\frac{(2 n+1) \pi}{2^{l}}\right) \\
& =\eta_{l, 0}\left(\theta-\theta_{l, n}\right) D_{\alpha}\left(\theta-\theta_{l, n}-\frac{\pi}{2^{l}}\right) \\
& =\eta_{l+1,0}\left(\theta-\theta_{l+1,2 n}\right) .
\end{aligned}
$$

and the properties 36, 38 and 39 imply that

$$
\begin{aligned}
\eta_{l+1,2 n+1}(\theta) & =\eta_{l, n}(\theta) D_{\alpha}\left(\theta-\frac{(2 n+1) \pi}{2^{l}}-\pi\right) \\
& =\eta_{l, 0}\left(\theta-\theta_{l, n}\right) D_{\alpha}\left(\theta-\theta_{l, n}-\frac{\pi}{2^{l}}-\pi\right) \\
& =\eta_{l+1,1}\left(\theta-\theta_{l+1,2 n}\right) \\
& =\eta_{l+1,0}\left(\theta-\theta_{l+1,2 n+1}\right) .
\end{aligned}
$$

thus the property 37 is verified for $l+1$

The combining of wavelet $\psi$ and function $\eta_{l, n}$, give a new wavelets whose Fourier transforms are

$$
\widehat{\psi}_{l, n}(\vec{k})=\widehat{\psi}(\vec{k}) \eta_{l, n}(\theta)
$$

Let $\psi_{\vec{b}, j, l, n}$ be wavelets $\psi_{l, n}$ translated by $\vec{b} \in \mathbb{R}^{2}$, dilated by $2^{j}$

$$
\psi_{\vec{b}, j, l, n}(\vec{x})=\frac{1}{2^{2 j}} \psi_{l, n}\left(\frac{\vec{x}-\vec{b}}{2^{j}}\right)
$$

Thus the Fourier transform of $\psi_{\vec{b}, j, l, n}$ is is defined by

$$
\begin{aligned}
\widehat{\psi}_{\vec{b}, j, l, n}(\vec{k}) & =\widehat{\psi}_{l, n}\left(2^{j} \vec{k}\right) e^{i \vec{k} \vec{b}} \\
& =\widehat{\psi}\left(2^{j} \vec{k}\right) \eta_{l, n}(\theta) \\
& =\widehat{\psi}\left(2^{j} \vec{k}\right) \eta_{l, 0}\left(\theta-\theta_{l, n}\right)
\end{aligned}
$$

The parameter $l$ determines a selectivity level. Indeed, the aperture of the cone in frequency space supporting $\widehat{\psi}_{l, n}$ is equal to $\frac{2 \pi}{2^{l}}+2 \alpha$, so the angular selectivity of these directional wavelets is proportional to $2^{l}$.

The continuous wavelet transform of the function $f \in L^{2}\left(\mathbb{R}^{2}\right)$ with wavelets $\psi_{\vec{b}, j, l, n}$ is the function

$$
\begin{aligned}
w_{\vec{b}, j, l, n} & =\left\langle\psi_{\vec{b}, j, l, n} \mid f\right\rangle \\
& =\int_{\mathbb{R}^{2}} \psi_{\vec{b}, j, l, n}^{*}(\vec{x}) f(\vec{x}) d^{2} \vec{x} \\
& =\int_{\mathbb{R}^{2}} \psi_{j, l, n}^{*}(\vec{x}-\vec{b}) f(\vec{x}) d^{2} \vec{x}
\end{aligned}
$$

Therefore, the continuous wavelet transform can be rewritten as a convolution product $\psi_{j, l, n} \star f(\vec{b})$, then the convolution theorem of Fourier transform imply that

$$
\widehat{w}_{., j, l, n}(\vec{k})=\widehat{\psi}_{j, l, n}(\vec{k}) \widehat{f}(\vec{k})
$$

The following proposition shows the possibility of using an adaptive selectivity decomposition:

THEOREM 2. For any function

$$
\tilde{l}: \quad \mathbb{R}^{2} \times \mathbb{Z} \rightarrow\{0,1, \ldots, L\}
$$

we obtain the following decomposition for any image $f \in L^{2}\left(\mathbb{R}^{2}\right)$

$$
f(\vec{x})=\left[\zeta_{., J} \star \phi_{J}\right](\vec{x})+\sum_{j=-\infty}^{J} \sum_{n=0}^{2^{\tilde{l}}-1}\left[w_{., j, \tilde{l}, n} \star \psi_{j, \tilde{l}, n}\right](\vec{x}),
$$

with

$$
\zeta_{\vec{b}, J}=\left\langle f \mid \phi_{\vec{b}, J}\right\rangle \quad \text { and } \quad w_{\vec{b}, j, \tilde{l}, n}=\left\langle f \mid \psi_{\vec{b}, j, \tilde{l}, n}\right\rangle .
$$

Proof. We have

$$
\begin{aligned}
w_{\vec{b}, j, \tilde{l}, n} & =\left\langle f \mid \psi_{\vec{b}, j, \tilde{l}, n}\right\rangle \\
& =\int_{\mathbb{R}^{2}} \psi_{\vec{b}, j, \tilde{l}, n}^{*}(\vec{x}) f(\vec{x}) d^{2} \vec{x} \\
& =\int_{\mathbb{R}^{2}} \psi_{j, \tilde{l}, n}^{*}(\vec{x}-\vec{b}) f(\vec{x}) d^{2} \vec{x} \\
& =\psi_{j, \tilde{l}, n}^{*} \star f(\vec{b})
\end{aligned}
$$

and

$$
\begin{aligned}
\zeta_{\vec{b}, j} & =\left\langle f \mid \phi_{\vec{b}, J}\right\rangle \\
& =\int_{\mathbb{R}^{2}} \phi_{\vec{b}, J}^{*}(\vec{x}) f(\vec{x}) d^{2} \vec{x} \\
& =\int_{\mathbb{R}^{2}} \phi_{J}^{*}(\vec{x}-\vec{b}) f(\vec{x}) d^{2} \vec{x} \\
& =\phi_{J}^{*} \star f(\vec{b})
\end{aligned}
$$

then the convolution theorem of fourier transform imply that

$$
\widehat{w}_{., j, \tilde{l}, n}(\vec{k})=\widehat{\psi}_{j, \tilde{l}, n}^{*}(\vec{k}) \widehat{f}(\vec{k})=\widehat{\psi}_{\tilde{l}, n}^{*}\left(2^{j} \vec{k}\right) \widehat{f}(\vec{k})
$$

and

$$
\widehat{\zeta}_{., J}(\vec{k})=\widehat{\phi}_{J}^{*}(\vec{k}) \widehat{f}(\vec{k})=\widehat{\phi}^{*}\left(2^{j} \vec{k}\right) \widehat{f}(\vec{k})
$$


Let $F_{J}$ and $K_{j, \tilde{l}, n}$ two function of $L^{2}\left(R^{2}\right)$ defends by

$$
F_{J}(\vec{x})=\left[\zeta_{., J} \star \phi_{J}\right](\vec{x})
$$

and

$$
K_{j, \tilde{l}, n}(\vec{x})=\left[w_{., j, \tilde{l}, n} \star \psi_{j, \tilde{l}, n}\right](\vec{x}),
$$

then the convolution theorem of fourier transform imply that

$$
\widehat{K}_{j, \tilde{l}, n}(\vec{k})=\widehat{\psi}_{j, \tilde{l}, n}(\vec{k}) \widehat{w}_{j, \tilde{l}, n}(\vec{k})=\widehat{\psi}_{\tilde{l}, n}\left(2^{j} \vec{k}\right) \widehat{w}_{j, \tilde{l}, n}(\vec{k})
$$

and

$$
\widehat{F}_{J}(\vec{k})=\widehat{\phi}_{J}(\vec{k}) \widehat{\zeta}_{J}(\vec{k})=\widehat{\phi}\left(2^{j} \vec{k}\right) \widehat{\zeta}_{J}(\vec{k}) .
$$

The properties 53 and 54 chow that

$$
\widehat{K}_{j, \tilde{l}, n}(\vec{k})=\widehat{\psi}_{\tilde{l}, n}\left(2^{j} \vec{k}\right) \widehat{\psi}_{\tilde{l}, n}^{*}\left(2^{j} \vec{k}\right) \widehat{f}(\vec{k})
$$

and

$$
\widehat{F}_{J}(\vec{k})=\widehat{\phi}\left(2^{j} \vec{k}\right) \widehat{\phi}^{*}\left(2^{j} \vec{k}\right) \widehat{f}(\vec{k}) .
$$

We shall now prove that

$$
\sum_{n=0}^{2^{\tilde{l}}-1} \widehat{K}_{j, \tilde{l}, n}(\vec{k})=\widehat{K}_{j, 0,0}(\vec{k})=\widehat{\psi}\left(2^{j} \vec{k}\right) \widehat{\psi^{*}}\left(2^{j} \vec{k}\right) \widehat{f}(\vec{k})
$$

According to the expression 31 and the property 33 we verify that

$$
D_{\alpha}^{2}(\theta)+D_{\alpha}^{2}(\theta-\pi)=1 .
$$

Hence, for any $l \in\{0, \ldots, L\}$

$$
\begin{aligned}
& \sum_{n=0}^{2^{\tilde{l}}-1} \widehat{K}_{j, \tilde{l}, n}(\vec{k}) \\
& =\sum_{n=0}^{2^{\tilde{l}}-1} \widehat{\psi}_{\tilde{l}, n}\left(2^{j} \vec{k}\right) \widehat{\psi}_{\tilde{l}, n}^{*}\left(2^{j} \vec{k}\right) \widehat{f}(\vec{k}) \\
& =\sum_{n=0}^{2^{\tilde{l}-1}-1}\left[\widehat{\psi}_{\tilde{l}, 2 n}\left(2^{j} \vec{k}\right) \widehat{\psi}_{\hat{l}, 2 n}^{*}\left(2^{j} \vec{k}\right)\right. \\
& \left.+\widehat{\psi}_{j, \tilde{l}, 2 n+1}\left(2^{j} \vec{k}\right) \widehat{\psi}_{j, \tilde{l}, 2 n+1}^{\star}\left(2^{j} \vec{k}\right)\right] \widehat{f}(\vec{k}) \\
& =\sum_{n=0}^{2^{\tilde{l}-1}-1}\left[\widehat{\psi}_{j, \tilde{l}-1, n}\left(2^{j} \vec{k}\right) \widehat{\psi}_{j, \tilde{l}-1, n}^{\star}\left(2^{j} \vec{k}\right) D_{\alpha}^{2}\left(\bar{\theta}_{n, \tilde{l}}\right)\right. \\
& \left.+\widehat{\psi}_{j, \tilde{l}-1, n}\left(2^{j} \vec{k}\right) \widehat{\psi}_{j, \tilde{l}-1, n}^{\star}\left(2^{j} \vec{k}\right) D_{\alpha}^{2}\left(\bar{\theta}_{n, \tilde{l}}-\pi\right)\right] \widehat{f}(\vec{k}) \\
& =\sum_{n=0}^{2^{\tilde{l}-1}-1} \widehat{\psi}_{j, \tilde{l}-1, n}\left(2^{j} \vec{k}\right) \widehat{\psi}_{j, \tilde{l}-1, n}^{\star}\left(2^{j} \vec{k}\right)\left[D_{\alpha}^{2}\left(\bar{\theta}_{n, \tilde{l}}\right)+D_{\alpha}^{2}\left(\bar{\theta}_{n, \tilde{l}}-\pi\right)\right] \widehat{f}(\vec{k}) \\
& =\sum_{n=0}^{2^{l-1}-1} \widehat{\psi}_{j, \tilde{l}-1, n}\left(2^{j} \vec{k}\right) \widehat{\psi}_{j, \tilde{l}-1, n}^{\star}\left(2^{j} \vec{k}\right) \widehat{f}(\vec{k}) \\
& =\sum_{n=0}^{2^{\tilde{l}-1}-1} \widehat{K}_{j, \tilde{l}-1, n}(\vec{k}) \text {. }
\end{aligned}
$$

Therefore,

$$
\sum_{n=0}^{2^{\tilde{l}}-1} \widehat{K}_{j, \tilde{l}, n}(\vec{k})=\widehat{K}_{j, 0,0}(\vec{k})=\widehat{\psi}\left(2^{j} \vec{k}\right) \widehat{\psi}^{*}\left(2^{j} \vec{k}\right) \widehat{f}(\vec{k})
$$

and the equation 27 yields

$$
\begin{aligned}
\widehat{f}(\vec{k}) & =\widehat{F}_{J}(\vec{k})+\sum_{j=-\infty}^{J} \widehat{K}_{j, 0,0}(\vec{k}) \\
& =\widehat{F}_{J}(\vec{k})+\sum_{j=-\infty}^{J} \sum_{n=0}^{2^{\tilde{l}}-1} \widehat{K}_{j, \tilde{l}, n}(\vec{k}) .
\end{aligned}
$$

This shows that

$$
\begin{aligned}
f(\vec{x}) & =F_{J}(\vec{x})+\sum_{j=-\infty}^{J} \sum_{n=0}^{2^{\tilde{l}}-1} K_{j, \tilde{l}, n}(\vec{x}) \\
& =\left[\zeta_{., J} \star \phi_{J}\right](\vec{x})+\sum_{j=-\infty}^{J} \sum_{n=0}^{2^{\tilde{l}_{-1}}}\left[w_{., j, \tilde{l}, n} \star \psi_{j, \tilde{l}, n}\right](\vec{x}) .
\end{aligned}
$$

\section{PULSE COUPLED NEURAL NETWORKS}

Pulse coupled neural networks (PCNN) were introduced as a simple model for the cortical neurons in the visual area of the cat's brain and monkey visual cortices . PCNN is a feedback network and each PCNN neuron consists of three parts: receptive field ,modulation field,and pulse generator. Unlike the most existing arti?cial neural networks, PCNN is based on iterative calculation and does not require any training process. The PCNN model applied in image processing tasks is generally a single-layer network with a 2-D array input. There is a one-to-one correspondence between input image pixels and PCNN neurons, so the number of neurons is equal to that of pixels. Each neuron is linked with its neighboring neurons for information transmission and coupling . The mathematical model of PCNN is described as follows [11, 12, 20]:

$$
F_{x y}(n)=S_{x y}
$$

$$
\begin{gathered}
L_{x y}(n)=e^{-\alpha_{L}} L_{x y}(n-1)+V_{L} \sum W_{x y, p q} Y_{x y, p q}(n-1) \\
U_{x y}(n)=F_{x y}(n)\left(1+\beta L_{x y}(n)\right)
\end{gathered}
$$

$$
Y_{x y}= \begin{cases}1, & \text { If } U_{x y}(n)>\theta_{x y}(n) \\ 0, & \text { otherwise. }\end{cases}
$$

$$
\theta_{x y}(n)=e^{-\alpha_{\theta}} \theta_{x y}(n-1)+V_{0} Y_{x y}(n-1)
$$

and

$$
T_{x y}(n)=T_{i j}(n-1)+Y_{x y}(n) .
$$

Here, $F, L, U, Y, T$ are the feeding input, the linking input, the internal activation, the output and dynamic threshold, respectively; $n$ is the discrete time step; pair $(x, y)$ represents neuron's position in 2D neural network; $S$ is the external stimulus (e.g., the intensity of pixel $(x, y)$ ). In addition, pair $p q$ represents the position with coordinates $(x+p, y+q)$. So $Y_{p q}$ 's are the outputs of neuron of position $(x, y)$ connects with its neighboring neurons. $T_{x y}(n)$ are often used to indicate the total firing times in $n$ iteration. The firing times are employed to represent image information. The SPCNN model is initialized as $Y_{x y}(0)=0, U_{x y}(0)=0$ and $\theta_{x y}(0)=0$. $n$ 
is the total iteration times. $\left(\alpha_{L}, \alpha_{\theta}\right)$ and $\left(V_{L}, V_{\theta}\right)$ are the time constants and normalizing constants for the linked input and dynamic threshold, respectively. The linking strength $\beta$ reflects the weight of linking field that plays a key role in fusion. Traditionally, $\beta$ is chosen according to experiences which is lack of self-adaptability.

\section{PROPOSED FUSION METHOD}

Supposed the source images $f_{A}$ and $f_{B}$ have been registered, the process of the proposed image fusion algorithm is as follows.

(1) Fix the largest scale $J \in \mathbb{N}^{*}$ and the highest selectivity level $L \in \mathbb{N}$.

(2) Compute the multiselectivity multiscale decomposition $(M M D)$ for the two source images $f_{A}$ and $f_{B}$

$$
M M D=\left\{\zeta_{\vec{b}, J}^{A}, \zeta_{\vec{b}, J}^{B} W_{\vec{b}, j, l, n}^{A}, W_{\vec{b}, j, l, n}^{B}\right\}
$$

where $\vec{b}=(x, y), 1 \leq j \leq J, 0 \leq l \leq L, 0 \leq n \leq 2^{l}-1$.

(3) According to PCNN model described from 63 to 68 we calculat the firing times $T_{x y}(N)$ of each neuron for the coefficients $\chi$ of $M M D$, where $N$ is the total number of iterations.

(4) The fused coefficients $\chi^{F}$ is obtained using the following

$$
\chi_{x y}^{F}= \begin{cases}\chi_{x y}^{A}, & \text { If } T_{x y}^{A}(N)>T_{x y}^{B}(N) ; \\ \chi_{x y}^{B}, & \text { otherwise. }\end{cases}
$$

(5) Determine $\widetilde{l}(j, \vec{x})$. One possibility is the choice of $\widetilde{l}(j, \vec{x})$ which offers the best match between the image and the wavelets,

$$
\widetilde{l}(j, \vec{x})=\arg \max _{l \in[0, L]} \max _{n \in\left[0,2^{l}-1\right]} \frac{W_{\vec{x}, j, l, n}^{F}}{\left\|\psi_{\vec{x}, j, l, n}\right\|}
$$

(6) Reconstruct the fused image $f_{F}$ by $\left\{\zeta_{\vec{b}, J}^{F},, W_{\vec{b}, j, l, n}^{F}\right\}$ and $\widetilde{l}(j, \vec{x})$ using 51

To quantitatively assess the performances of different methods, five widely recognized objective fusion metrics are applied in our experiments, shannon entropy $(E N)$, average gradient $(A G)$, Standard Deviation $(S D)$, mutual information $M I$ and $Q A B / F$.

Shannon entropy $E N$ quantifies the richness of information in the fused image. The larger the $E N$ value is, the more abundant the information amount of the fusion image is. The shannon entropy of a fused image was calculated according to

$$
E N=-\sum_{i=0}^{255} P_{i} \log \left(P_{i}\right)
$$

where $P_{i}$ is the probability of gray level $i$ in the fused image

Average gradient $A G$ reflects the variance of the gray value which could be used to evaluate the clarity of one image. The higher value of gradient value denotes that the image is clearer and the fusion performance is better. The definition is shown as

$$
\begin{gathered}
A G=\frac{1}{\left(l_{x}-1\right)\left(l_{y}-1\right)} \sum_{i=1}^{l_{x}} \sum_{j=1}^{l_{y}} \sqrt{\frac{\mu_{x}(i, j)+\mu_{y}(i, j)}{2}} \\
\mu_{x}(i, j)=\left(f_{F}(i, j)-f_{F}(i+1, j)\right)^{2}
\end{gathered}
$$

$$
\mu_{y}(i, j)=\left(f_{F}(i, j)-f_{F}(i, j+1)\right)^{2}
$$

$f_{F}(i, j)$ is the pixel value of the fused image at position $(i, j) ; l_{x}$ $l_{y}$ decide the area (the biggest time is $3 \times 3$ ).

Standard deviation $S D$ measures the degree that the data points deviate from the mean. The standard deviation was calculated according to

$$
S D=\sqrt{\frac{\sum\left(f_{F}(i, j)-\bar{f}_{F}\right)^{2}}{T-1}}
$$

where $f_{F}(i, j)$ is the pixel value of the fused image at the position of $(i, j) ; f_{F}$ is the average pixel value; $T$ is the number of the pixel of an image. A larger standard deviation means a better visible effect.

Mutual information $M I$ essentially computes how much information from source images is transferred to the fused image. With the increase of the value of mutual information, fused image can get richer information from the source image.

$$
M I=M I_{F A}+M I_{F B}
$$

Where $M I_{F A}$ or $M I_{F B}$ is denotes the mutual information between the Fused image and original image, which is defined as follows

$$
M I_{F A}=\sum_{i=0}^{N} \sum_{J=0}^{M} P_{F A}(i, j) \log \frac{\left.P_{F A}(i, j)\right)}{P_{F}(i, j)+P_{A}(i, j)}
$$

$P_{F A}(i, j)$ is the joint histogram of the fused image $f_{F}$ and the source image $f_{A}$ |21].

QAB/F computes and measures the amount of edge information transferred from the source images to the fused images using a Sobel edge detector to calculate strength and orientation information at each pixel in both source and the fused images. The larger the value is, the more edge information is transformed to the fused image. The definition of $Q A B / F$ is defined as

$$
\begin{gathered}
\frac{\sum_{i=0}^{N} \sum_{J=0}^{M}\left(Q_{i j}^{A F} w_{i j}^{A}+Q_{i j}^{B F} w_{i j}^{B}\right)}{\sum_{i=0}^{N} \sum_{J=0}^{M}\left(w_{i j}^{A}+w_{i j}^{B}\right)} \\
Q_{i j}^{A F}=Q_{g i j}^{A F}+Q_{i j}^{\alpha A F} \\
Q_{i j}^{B F}=Q_{g i j}^{B F}+Q_{i j}^{\alpha B F}
\end{gathered}
$$

$Q_{g i j}^{. F}$ and $Q_{i j}^{\alpha . F}$ denote the edge strength and orientation preservation values, respectively [22]. The larger value means the better fusion performance.

\section{EXPERIMENTAL RESULTS}

In this section, we use proposed method to fuse the medical images, we conduct experiments on six pairs of multimodal medical images, which include, three pairs of MR and CT images, and three pair of T1-weighted MR (MR-T1), T2-weighted MR (MR-T2) and PET images. Parameters of PCNN is set, $\alpha^{L}=0.06931, \alpha^{\theta}=0.2$, $\beta=0.2, V_{L}=1.0, V_{\theta}=20, W=\left(\begin{array}{ccc}0.707 & 1 & 0.707 \\ 1 & 0 & 1 \\ 0.707 & 1 & 0.707\end{array}\right)$, and the 
Table 1. Objective assessment of different methods on four categories of medical image fusion problems

\begin{tabular}{ccccccc}
\hline images & Methods & EN & AG & SD & MI & QAB/F \\
\hline \hline CT and MR & & & & & & \\
\hline \hline & DWT-PCNN & 5.547 & 8.156 & 68.2251 & 4.354 & 0.6188 \\
& NSCT-PCNN & 6.4040 & 8.6349 & 69.2021 & 4.5821 & 0.6188 \\
& NSST-PCNN & 6.3966 & $\underline{8.6632}$ & 69.1797 & 4.6531 & 0.6315 \\
& Proposed Method & $\underline{6.8202}$ & 8.6454 & $\underline{69.2670}$ & $\underline{4.9706}$ & $\underline{0.6521}$ \\
\hline \hline CT and MR & & & & & & \\
\hline \hline & DWT-PCNN & 4.5684 & 7.0387 & 70.2581 & 3.0254 & 0.4231 \\
& NSCT-PCNN & 5.5821 & $\underline{7.6066}$ & 72.8854 & 3.4747 & 0.5060 \\
& NSST-PCNN & 5.5249 & 7.5736 & $\underline{73.0768}$ & 3.5949 & 0.5155 \\
& Proposed Method & $\underline{5.6978}$ & 7.5891 & 72.9821 & $\underline{3.6769}$ & $\underline{0.5551}$ \\
\hline \hline MRT1 and MRT2 & & & & & & \\
\hline \hline & DWT-PCNN & 4.2148 & 8.5478 & 71.2025 & 1.1258 & 0.4518 \\
& NSCT-PCNN & 5.2207 & 9.9223 & 74.0258 & 2.5367 & 0.5166 \\
& NSST-PCNN & 5.1852 & $\underline{10.1272}$ & $\underline{75.6418}$ & 2.5785 & 0.5252 \\
& Proposed Method & $\underline{5.2880}$ & 10.0846 & 75.4195 & $\underline{2.6561}$ & $\underline{0.5840}$ \\
\hline \hline MR and PET & & & & & & \\
\hline \hline
\end{tabular}
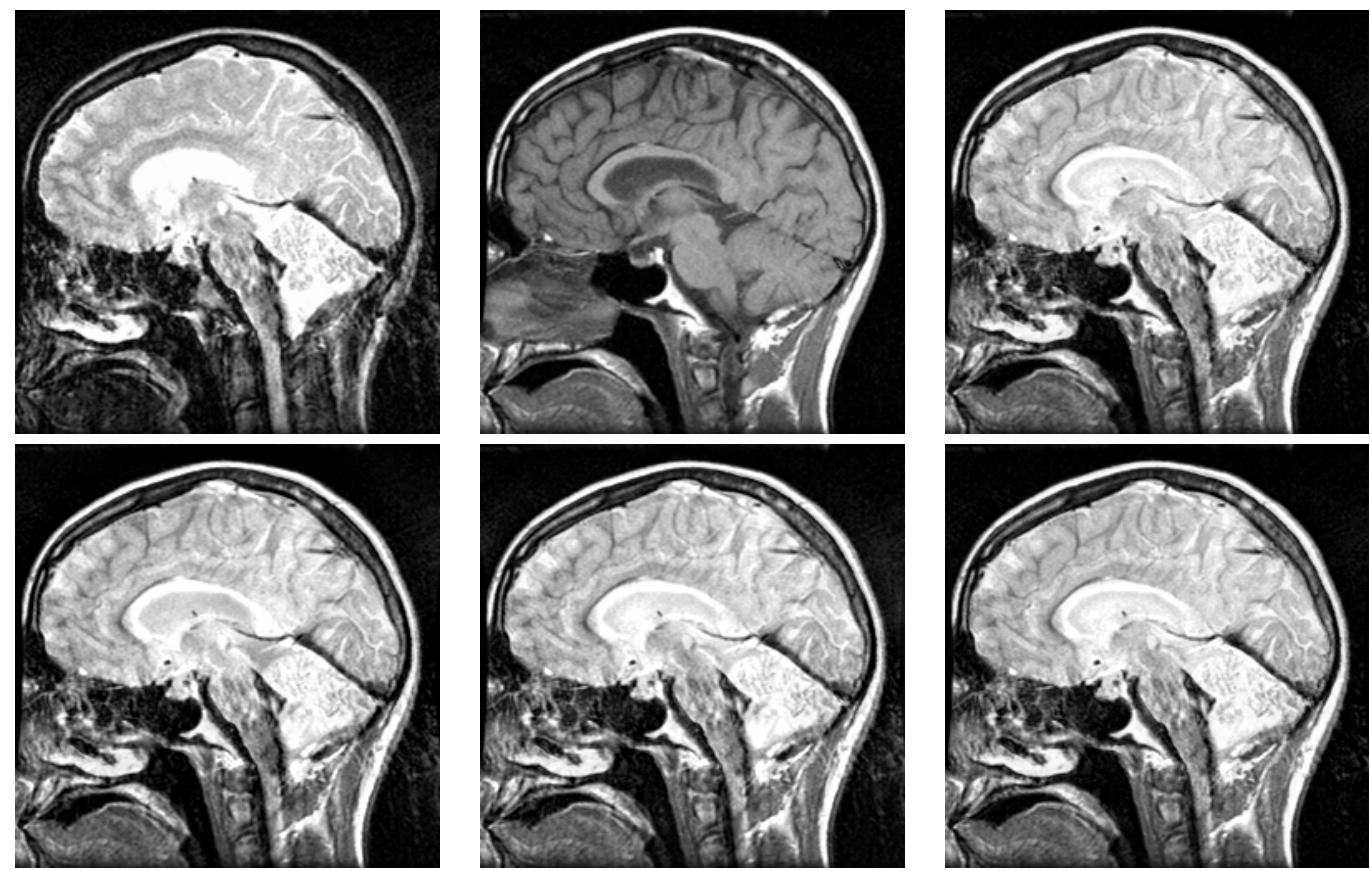

Fig. 1. Example of CT and MR images fusion obtained by different methods. From top left, clockwise: CT, MR, DWT-PCNN, NSCT-PCNN, NSST-PCNN and proposed method.

maximal iterative number is $N=200$. We compare the fusion results of the proposed framework with the following methods on the same dataset.

- Method 1 DWT-PCNN: Image fusion method based on pulse couple neuron network in discrete wavelet transform Domain with basis $\mathrm{db} 2$.
- Method 2 NSCT-PCNN: Image fusion method based on pulse couple neuron networkin the Nonsubsampled Contourlet Transform Domain.

- Method 3 NSST-PCNN: Image fusion method based on pulse couple neuron network in the nonsubsampled shearlet transform Domain. 

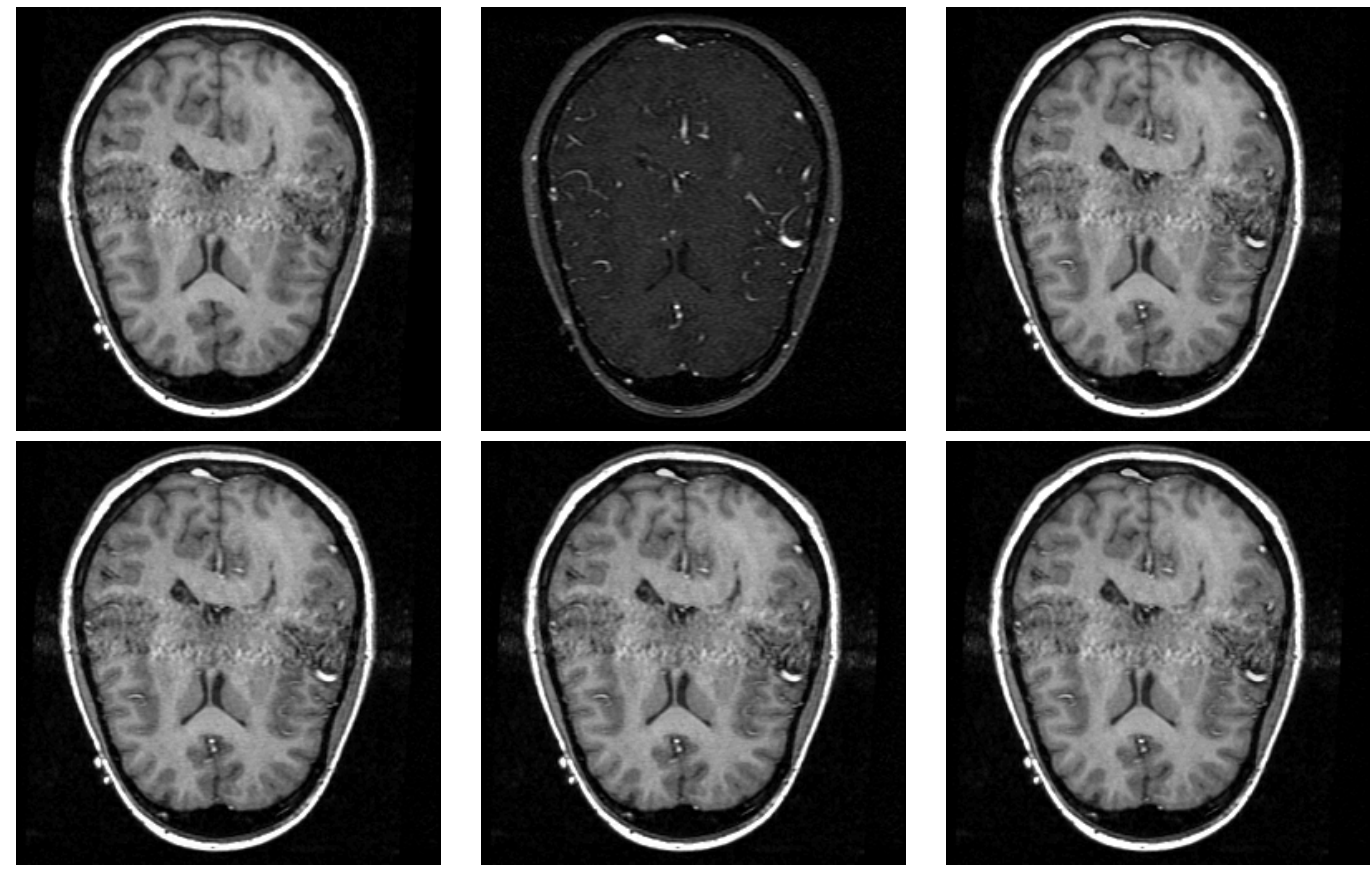

Fig. 2. Example of MR-T1 and MR-T2 images fusion obtained by different methods. From top left, clockwise: MR-T1, MR-T2, DWT-PCNN, NSCT-PCNN, NSST-PCNN and proposed method.
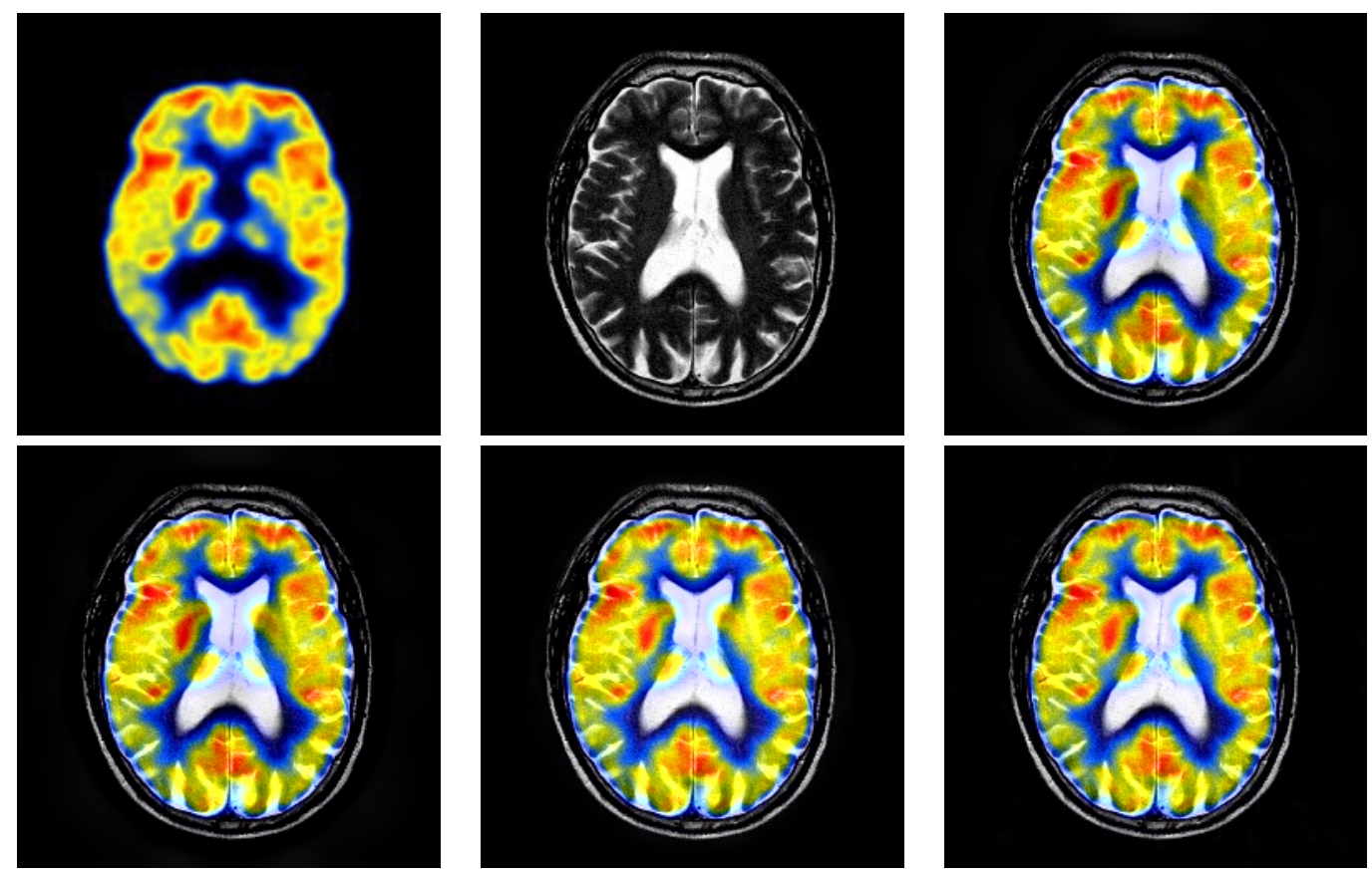

Fig. 3. Example of PET and MR images fusion obtained by different methods. From top left, clockwise: PET, MR, DWT-PCNN, NSCT-PCNN, NSST-PCNN and proposed method.

Table 1 shows evaluation results of four methods. The $E N$ of the fused image obtained by the proposed method is maximum, which means that the fused image contains the largest amount of information and has relatively better fusion result than others. The $M I$ and $Q A B / F$ achieve the best result of all, which illustrates that the fused image based on the proposed method extracts more information from the original images and has the best contrast and the notable target. 
From the visual analysis of Figures 1,2 and 3, it is observed that the proposed framework could successfully preserve both the feature information of the CT images (e.g., the bony structures) and the PET images (e.g., high metabolic areas).

\section{CONCLUSION}

In this paper, we introduced a new medical image fusion method based on a adaptive selectivity representation, which can capture the different structures of the geometry of the image. The fused images processed by this method have improved fusion quality compared with those produced by other methods in terms of better balance between feature information and information for soft tissues, and better preserving of functional information.

\section{REFERENCES}

[1] HM. Yin, X. Liu, Y. Liu, and X. Chen. Medical image fusion with parameter-adaptive pulse coupled neural network in nonsubsampled shearlet transform domain. IEEE Transactions on Instrumentation and Measurement, 68(1):49-64, Jan 2019.

[2] A. P. James and B. V. Dasarathy. Medical image fusion: A survey of the state of the art. Inf. Fusion, 19:419, 2014

[3] D. P. Bavirisetti, G. Xiao, and G. Liu. Multi-sensor image fusion based on fourth order partial differential equations. Proceedings of the International Conference on Information Fusion, page 19, 2017.

[4] Zhao, G. Cui, X. Gong, Y. Zang, S. Tao, and D. Wang. Fusion of visible and infrared images using global entropy and gradient constrained regularization. Infrared Physics and Technology, 81:201209, 2017.

[5] J. Ma, C. Chen, C. Li, and J. Huang. Infrared and visible image fusion via gradient transfer and total variation minimization. Information Fusion, 31:100109, 2016.

[6] J. Zhao, Y. Chen, H. Feng, Z. Xu, and Q. Li. Infrared image enhancement through saliency feature analysis based on multi-scale decomposition. Infrared Physics and Technology, 62:8693, 2014.

[7] X. Zhang, Y. Ma, F. Fan, Y. Zhang, and J. Huang. Infrared and visible image fusion via saliency analysis and local edge-preserving multi-scale decomposition. JOSA A, 34(8):14001410, 2017.

[8] H. Li, B. S. Manjunath, and S. K. Mitra. Multisensor image fusion using the wavelet transform. Graph. Models Image Process, 57(3):235245, 1995.

[9] P. J. Burt and E. H. Adelson. he laplacian pyramid as a compact image code. IEEE Trans. Commun, 31(4):532-540, 1983.

[10] F. Yong and L. Sheng-Peng. Infared image fusion algo- rithm based on contourlet transform and improved pulse coupled neural networks. China Patent 1873693A, 2006.

[11] X.-B. Qu, J.-W. Yan, H.-Z. Xiao, and Z.-Q. Zhu. Image fusion algorithm based on spatial frequency-motivated pulse coupled neural networks in nonsubsampled contourlet transform domain. Acta Autom, 34(12):15081514, 2008.

[12] G. Bhatnagar, Q. M. J. Wu, and Z. Liu. Directive contrast based multimodal medical image fusion in nsct domain. IEEE Trans. Multimedia, 15(5):10141024, 2013.
[13] G. Guorong, X. Luping, , and F. Dongzhu. Multi-focus image fusion based on non-subsampled shearlet transform. IET Image Process, 7(6):633639, 2013.

[14] J-P. Antoine, R. Murenzi, P. Vandergheynst, and S.T. Ali. Two-dimensional wavelets and their relatives. Cambridge University Press, 2004.

[15] J-P. Antoine, P. Carrette, R. Murenzi, and B. Piette. Image analysis with two-dimensional continuous wavelet transform. Signal Processing, 31:241272, 1993.

[16] S. Mallat. A wavelet tour of signal processing. Academic Press, 1998.

[17] M. El Aallaoui, A. El Bouhtouri, and A. Ayadi. Adaptive selectivity representation: Design, and applications. Int. J. Wavelets, Multiresolution and Information Processing (ijwmip), 7:89-113, 2009.

[18] S. Mallat. A wavelet tour of signal processing. Academic Press, 1998.

[19] I. Daubechies. Orthonormal bases of compacty supported wavelets. on Pure and appl.Math, 241:909-996., 1988.

[20] Y. Chen, S. K. Park, Y. Ma, and R. Ala. A new automatic parameter setting method of a simplified pcnn for image segmentation. IEEE Trans. Neural Netw, 22(6):880892, 2011.

[21] M. Hossny, S. Nahavandi, D. Creighton, and A. Bhatti. Image fusion performance metric based on mutual information and entropy driven quadtree decomposition. Electron. Lett, 46(18):12661268, 2010.

[22] Z. Song, H. Jiang, and S. Li. A novel fusion framework based on adaptive pcnn in nsct domain for whole-body pet and ct images. Hindawi Computational and Mathematical Methods in Medicine, 2017:1-9, 2017. 